made somehow infected and lysogenized $E$. coli in someone's gut as the result of an accident? This possibility, remote though it may seem to be, can hardly be ignored, and it will be most interesting to learn what criteria the group adopts when it decides whether or not the scientific information that might be obtained by continuing the experiment justifies the risk. Perhaps those involved will decide that the game is not worth the candle.

Whatever the outcome of any heart searchings the expertise with which the group have manufactured the hybrid DNA commands universal admiration. They have used the $R_{I}$ restriction endonuclease to convert circular SV40 DNA into linear molecules by cleavage at a unique site. They then removed a few bases from the $5^{\prime}$ ends of the linear chains with $\lambda$ exonuclease and added homodeoxypolymeric extensions (either poly (dT) or poly (dA)) to the $3^{\prime}$ ends of the two chains of this linear SV40 DNA. By the same procedure they prepared linear $\lambda d v g a l$ DNA with $3^{\prime}$ homodeoxypolymeric extensions. When the SV40 DNA and the $\lambda d v g a l$ DNA carrying complementary $3^{\prime}$ extensions are annealed they form circular duplex molecules, the gaps and nicks in which were sealed with $E$. coli DNA polymerase $I$ and DNA ligase to form covalently closed hybrid circular DNA molecules. And, of course, if this procedure is used with SV40 DNA alone the product is circular dimeric SV40 DNA.

As Berg and his colleagues observe, this procedure for covalently joining pairs of DNA molecules should be generally applicable with only minor modifications, and the exploitation of homopolymeric extensions means that quite unrelated DNAs can be joined. On the other hand these homopolymeric (dA.dT) tracts in the joined molecules may prove to be a hindrance when it comes to testing the biological activity of such hybrid DNAs, but that remains to be seen.

\section{PERCEPTION \\ Size Constancy}

from our Experimental Psychology Corresponden $_{\mathrm{t}}$

Is adaptation to specific stimuli the psychologist's equivalent of the neurophysiologist's most successful of instruments, the microelectrode? If so, it offers one of the most important methods for approaching an explanation of perception in terms of the workings of the nervous system. It is appropriate therefore that in the new journal Perception $(1,111 ; 1972)$ one of the more interesting articles (by Blakemore, Garner and Sweet of the University of Cambridge) should describe an applica- tion of this technique to the problem of size constancy.

The idea that neurones in sensory systems are each tuned to respond to a particular kind of event in the world is a very old one, going back at least to Descartes. More recently Sherrington described the event which a particular neurone seems designed to detect as its "adequate stimulus", and much of the success of modern neurophysiology can be attributed to the ability of the microelectrode to monitor the electrical activity of a single neurone; this provides an opportunity to discover its adequate stimulus. The discovery that cells in fairly peripheral parts of sensory systems are tuned not just to simple parameters such as sense modality or position on the body surface, but to fairly complex patterns, indicates that neuronal networks have an abstracting power which may be amenable to exact analysis. It further offers the possibility of explaining how the brain detects not just changes of energy on the receptor surface, but events of importance to the animal in the outside world. By continually showing an alert human subject a stimulus thought to be adequate and specific for a particular type of neurone, one can perhaps fatigue (that is, adapt) the same neurones from which one can record. This affords a means for investigating the activity of these cells and their effect on perception in man without recourse to anaesthetics, surgery or any of the other encumbrances of physiological experimentation.

One perceptual effect of viewing an object in conditions under which its distance can be judged is that size constancy obtains; the object looks to be of a size appropriate to its existence in the real world, not a size dependent only on the extent of its retinal image. The size seen depends on the brain analysing not just its retinal size but also its distance. Adapting the observer to a sinusoidal grating stimulus (a set of stripes in which brightness varies sinusoidally across the grating), which Blakemore et al. argue is specifically detected by neurones involved in size estimation, might then give one of two results. Either such an adaptation would depress sensitivity in those neurones sensitive to a grating that width of stripe on the retina, or, if the neurones in question have already scaled the grating for its size in the real world, the depression of sensitivity would be in neurones sensitive to a particular apparent width of stripes. By adapting to stripes subtending a given angle at the retina, and testing with gratings at a different distance, Blakemore et al. found that the depression of sensitivity occurred for test gratings of the same retinal size as the adapting grating.

The effect is surprising because as one adapts to, say, a grating with wide stripes far away, it looks as if it has

\title{
Evidence of Human Cancer Viruses
}

IN Nature New Biology next week (November 15) two groups, those led by Spiegelman and by Gallo, report further experiments which they believe strongly support the idea that human RNA tumour viruses, analogous to the viruses which cause sarcomas and leukaemias in mice and chickens, not only exist but also play a part in the development of these diseases in man. Spiegelman's group have during the past several months used RNA/DNA hybridization techniques to probe for RNA molecules in human leukaemic cells which have homology with the RNA in mouse leukaemia virus particles. They believe they have found such RNA molecules and they now report that they have detected a complex of 70S RNA and RNA-dependent DNA polymerase in the cytoplasm of 95 per cent of the samples of human leukaemic cells which they have examined. Furthermore they found that when this complex was incubated with appropriate substrates, DNA was made which hybridized to the RNA from mouse leukaemia virus particles but failed to hybridize to the RNAs of mouse mammary tumour virus particles and avian myeloblastosis virus particles.

Two of the diagnostic properties of the RNA tumour viruses of animals are the presence of reverse transcriptase and of 70S RNA, and so Spiegelman and his colleagues clearly think that they may have detected traces of a human leukaemia virus. Gallo and his colleagues also argue that they may be on the tracks of such viruses. They have found a reverse transcriptase activity associated with a particulate fraction from the cytoplasm of human leukaemic white blood cells. This activity, which has been partially purified, transcribes added avian myeloblastosis virus RNA into DNA, and because of its response to various synthetic RNA templates Gallo and his colleagues conclude that this activity is distinct from other cellular nucleic acid polymerases but is similar to the reverse transcriptase that occurs in animal and avian RNA tumour virus particles.

Obviously both these sets of experiments are consonant with the idea that human tumour virus particles exist, and they will no doubt stimulate further efforts to isolate these putative viruses in a pure and infectious form. 